\title{
Kinematic Analysis of a Planer Robot Using Artificial Neural Network
}

\author{
Jolly Shah", S.S.Rattan*, B.C.Nakra** \\ *ME Department, NIT, Kurukshetra, 136118, India. \\ ** ME Department, ITM University, Gurgaon,122001, India
}

\begin{tabular}{|c|c|}
\hline Article Info & ABSTRACT \\
\hline Article history: & Automatic control of the robotic manipulator involves study of kinematics \\
\hline Received May 31, 2012 & kinematics of 3-DOF planer robotic manipulator with all revolute joints. In \\
\hline Revised Aug 10, 2012 & this study the Denavit- Hartenberg (D-H) model is used to model robot links \\
\hline Accepted Aug 17, 2012 & $\begin{array}{l}\text { and joints. Also forward and inverse kinematics solution has been achieved } \\
\text { using Artificial Neural Networks for 3-DOF robotic manipulator. It shows }\end{array}$ \\
\hline Keyp & and has zero error. \\
\hline
\end{tabular}

Artificial Neural Network

Forward Kinematics

Inverse Kinematics

Robotic Manipulator

Robotics

Copyright (c) 2012 Institute of Advanced Engineering and Science. All rights reserved.

\section{Corresponding Author:}

Jolly Shah,

Departement of Mechanical Engineering,

National Institute of Technology,

Kurukshetra, 136118,India.

Email: jollyshah80@yahoo.com

\section{INTRODUCTION}

A kinematic equation provides the relationship between the joint displacement and the resulting endeffector position and orientation.

\subsection{Forward and inverse kinematics}

The problem of finding the end-effector position and orientation for a given set of joint displacements is referred to as the forward kinematics problem. That is, the forward kinematics problem allows one to specify in a unique manner the relationship between the ( $\mathrm{n} \times 1)$ joint vector $\theta$ and the $(\mathrm{m} \times 1)$ Cartesian vector $x$ as: $\mathrm{x}(\mathrm{t})=\mathrm{f}(\theta(\mathrm{t}))$

Where $\mathrm{f}$ is the function defining the forward kinematic relation of the manipulator. Normally, the forward Kinematic equation can be obtained from the spatial geometry of the manipulator or by solving certain matrix algebraic equations. As the number of degrees of freedom (n) increases, the kinematic equation becomes more complex. Hence, the amount of computation required to compute the end-effectors position can become quite large.

The inverse kinematics problem consists of the determination of the joint variables corresponding to a given end-effectors orientation and position and is given by the equation,

$\theta(\mathrm{t})=\mathrm{f}^{-1}(\mathrm{x}(\mathrm{t}))$

As compared to forward kinematics problem, the inverse kinematics problem is much more complex due to the following reasons: 
- In the joint variables, the equations to be solved are in general non-linear form and, thus, mostly, it is not always possible to find a closed form solution.

- Multiple solutions may exist.

- Infinite solutions may also exist, example - in redundant robot manipulators.

In order to find the solutions to the inverse kinematics, problem can be formulated either by finding a closed form solution using algebra or geometry or by finding a numerical solution by some successive approximation algorithm.[1,2,3,5,8]

\subsection{Artificial neural network}

It is an area of computer science, which is basically concerned with designing intelligent computer systems. It is mostly inclined towards those systems that exhibit the characteristics we associate with intelligence in human behavior. In other words, Artificial Intelligence is a branch of computer science that is concerned with the automation of intelligent behavior. In order to build intelligent systems, it includes various technologies in its domain such as expert systems, neural networks, fuzzy logic, cellular automata and probabilistic reasoning.

"A Neural Network is a massive parallel distributed processor made up of simple processing units has a tendency to acquired the knowledge from its environment through a learning process, store the knowledge and making it available for us.”[4]

Artificial neural networks are widely used as an effective approach for handling non-linear and noisy data[7], especially in situations where the physical processes relationships are not fully understood and they are also particularly well suited to modelling complex systems on a real-time basis. Basic structure of any artificial neural network is shown in Figure 1. Also basic neural network architecture is shown in Figure 2.

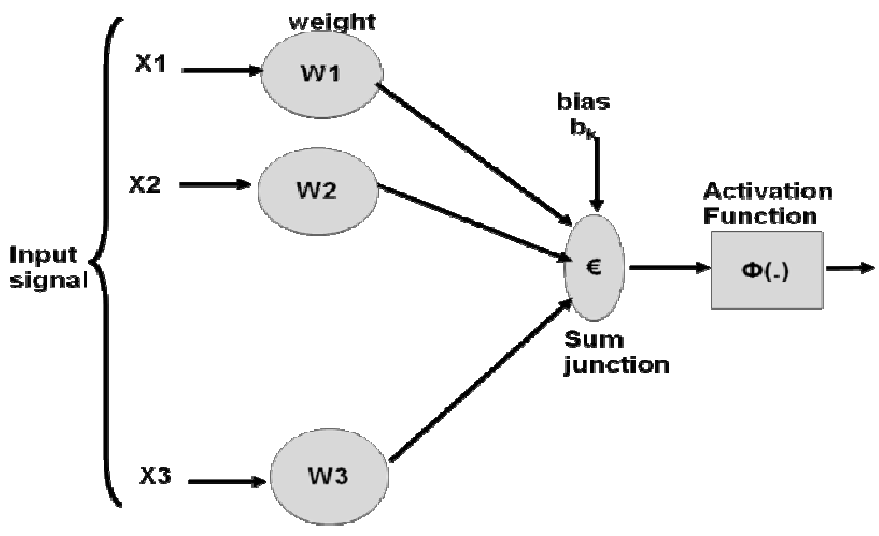

Figure 1. Representation of ANN Structure

In mathematical terms, we may describe a neuron $K$ by writing the following terms:

$U k=(w 11 x 1+w 12 x 2+\ldots w k m x m)$

and

$Y k=\phi(u k+b k)$

Where, $x 1, x 2, x m$ are the input signals,

$W k j$ are the synaptic weights of neuron $K$,

$U k$ is the linear combiner output due to the input signals,

$B k$ is the bias,

$\phi($.$) is the activation function$

Here the work focuses on a popular feed forward model of neural networks.

In this model a set of inputs are applied to the network, and multiplied by a set of connection weights.

All of the weighted inputs to the neuron are then summed and an activation function is applied to the summed value. This activation level becomes the neuron's output and can be either an input for other neurons, or an output for the network. Learning in this network is done by adjusting the connection weights based upon training vectors (input and corresponding desired output). When a training vector is presented to a neural net, the connection weights are adjusted to minimize the difference between the desired and actual output. 


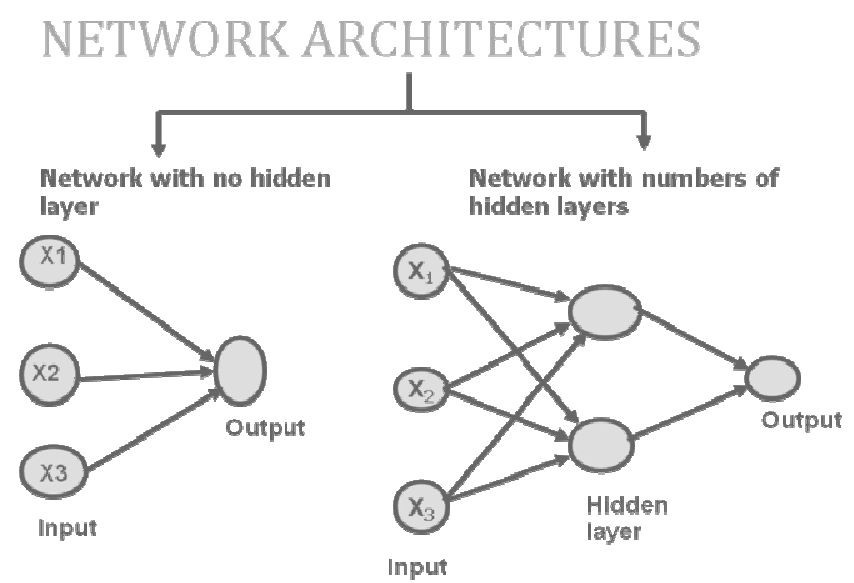

Figure 2. Artificial Neural Network Architecture

Artificial Neural networks provide a fast, method of learning to produce a set of output states given a set of input states. The advantages of using an artificial neural network approach in robotics applications such as in the forward kinematics problem are given below and also discussed in [6]:

- Programming required is not complex;

- The time to obtain a solution is independent of the number of manipulate or degrees of freedom;

- Fault tolerance;

- Capability of operating in real-time.

\section{RESEARCH METHOD}

In this study, the Denavit-Hartenberg (D-H) approach has been used for modeling the 3-DOF robotic manipulator. The forward and inverse kinematic solutions have been deduced using kinematic equations for 3-DOF robotic manipulator as shown in Figure 3.

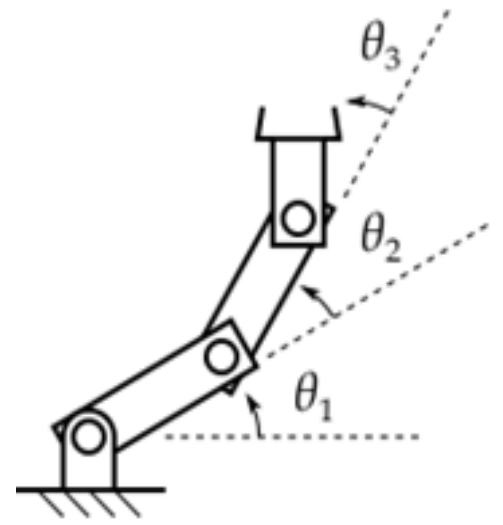

Figure 3. Representation of 3-DOF robotic manipulator

By MATLAB programming a data set has been generated. Data obtain in form of set of positionorientation of robot is used to train an artificial neural network, feed forward neural network is used and trained by using MATLAB toolbox.

\section{RESULTS AND ANALYSIS}

\subsection{Work-volume of 3-DOF robotic manipulator}

Denavit-Hartenberg (D-H) representation is used to model the joints of 3-DOF robotic manipulator, as discussed in [1,2]. The D-H parameters are described as di (distance on the $z$ axis between two successive 
normals), $a i$ (distance between joints along $x$ axis), $\theta i$ (rotation about the $z$ axis on $x y$ plane) and $\alpha i$ (joint twist). These parameters describe the location of a robot link frame $F i$ (a joint) from a preceding link frame $F i-1$ (previous joint) through the sequence of translations and rotations.

The D-H parameters for 3-DOF robotic manipulator are given in Table 1.

Table 1. D-H Parameters For 3-Dof Robotic Manipulator

\begin{tabular}{ccccc}
\hline Joint & $\theta\left(^{0}\right)$ & $\alpha_{i}\left(^{0}\right)$ & $a_{i}(\mathrm{~mm})$ & $d_{i}(\mathrm{~mm})$ \\
\hline 1 & 0 & -90 & 0 & 226 \\
2 & -90 & 0 & 179 & 0 \\
3 & 0 & 0 & 177 & 0 \\
\hline
\end{tabular}

In this paper, a 3-DOF robotic manipulator has been considered with link lengths $L 1=226 \mathrm{~mm}$, $L 2=179 \mathrm{~mm}$ and $L 3=177 \mathrm{~mm}$, respectively, as shown in fig.1, where, $L 1, L 2$ and $L 3$ are link lengths and $\theta 1$, $\theta 2$ and $\theta 3$ are joint angles, respectively. In forward kinematics solution, if the link lengths and angles are known, then it is possible to calculate the co-ordinates of the end-effectors of the robot at any instant. The MATLAB program has been run to find the co-ordinates $(x, y, z)$ of the 3-DOF robotic manipulator and which has found out to be $(0,177,325) \mathrm{mm}$, respectively at the home position.

The forward kinematics equations for 3-DOF robotic manipulator have been deduced as:

$$
\begin{aligned}
x & =L_{1} \times \cos \theta_{1}+L_{2} \times \cos \left(\theta_{1}+\theta_{2}+\theta 3\right) \\
y & =L_{1} \times \sin \theta_{1}+L_{2} \times \sin \left(\theta_{1}+\theta_{2}+\theta 3\right) \\
\emptyset & =\theta_{1}+\theta_{2}+\theta_{3}
\end{aligned}
$$

Using the forward kinematic equations of the robotic manipulator, all the $(x, y)$ co-ordinates of the 3DOF robotic manipulator have been generated and the work-volume has been plotted for all possible $\theta 1, \theta 2$, $\theta 3$ combinations as represented in Figure 4. [9]

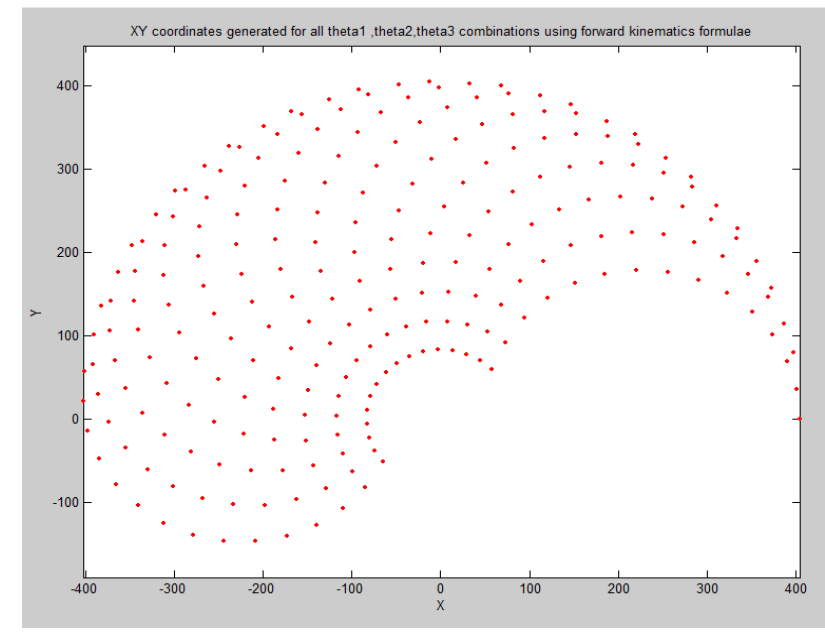

Figure 4. Work-volume of 3-DOF robotic manipulator.

\subsection{Forward kinematics using artificial neural network}

Data obtain in form of set of position-orientation of robot using MATLAB programme is used to train neural network. Feed Forward neural network is used and trained by using MATLAB toolbox. Training graph is as shown in fig.5. X-axis and $\mathrm{Y}$-axis of fig.5 represents number of epochs and performance respectively. Also comparison between actual target points and predicted target points are shown in fig.6, X co-ordinate values and Y-coordinate values are represented by blue and green colours respectively. Also dot represents actual targets and dash represents predicted targets values. 


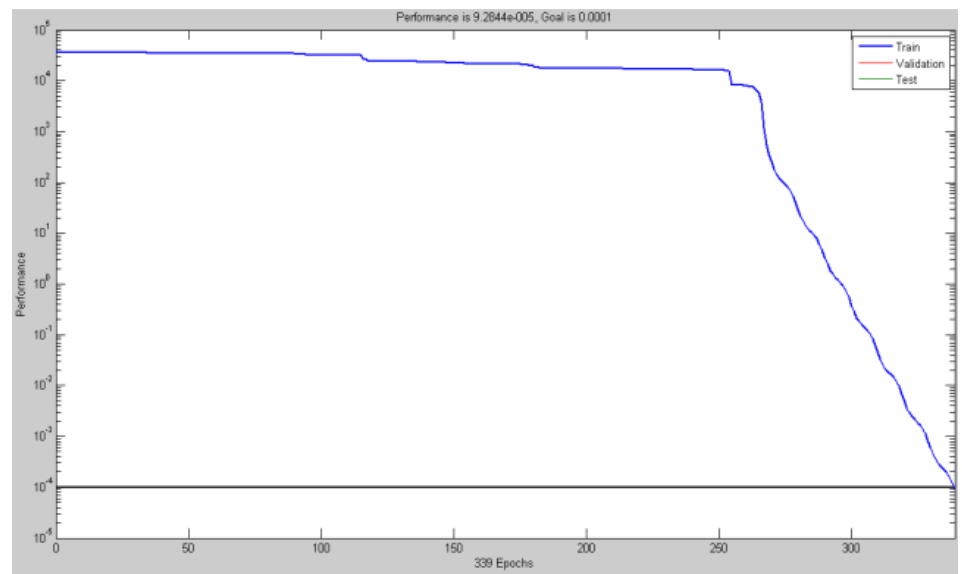

Figure 5.Training graph for forward kinematics using ANN

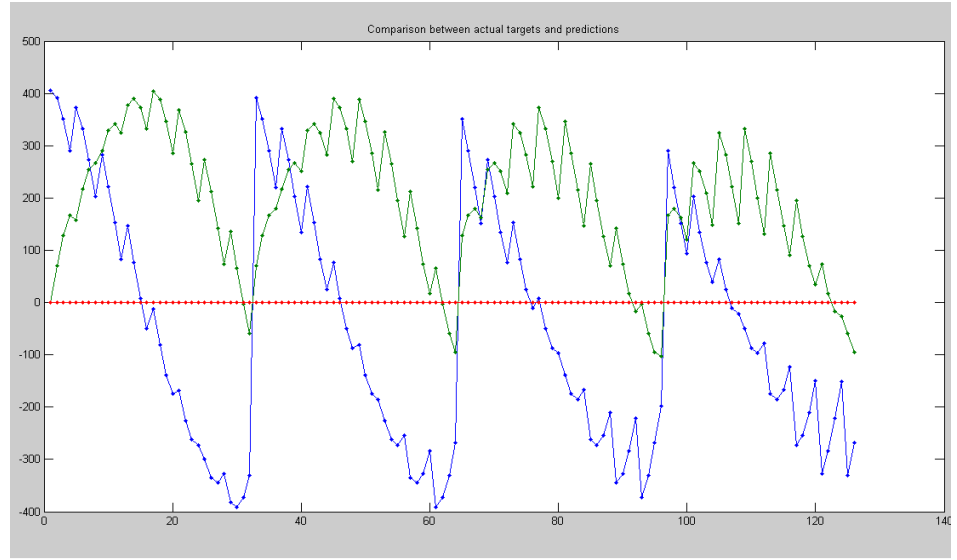

Figure 6.Comparison between actual and predicted targets for forward kinematic.

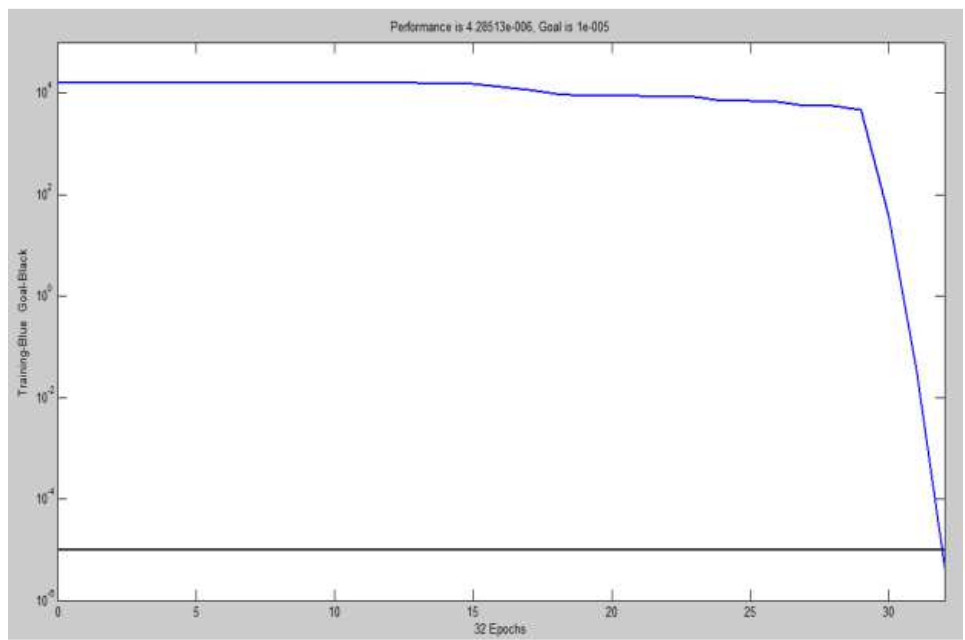

Figure 7. Training graph for inverse kinematics using ANN

\subsection{Inverse kinematics using artificial neural network}

Data obtain in form of set of orientation-position of robot using MATLAB programme is used to train neural network. Feed Forward neural network is used and trained by using MATLAB toolbox. Training 
graph is as shown in fig.7. $\mathrm{X}$-axis and $\mathrm{Y}$-axis of fig.7 represents number of epochs and performance respectively Also comparison between actual target points and predicted target point are shown in fig.8 [10] $\mathrm{X}$ co-ordinate values and $\mathrm{Y}$-coordinate values are represented by blue and green colours respectively. Also dot represents actual targets and dash represents predicted targets values.

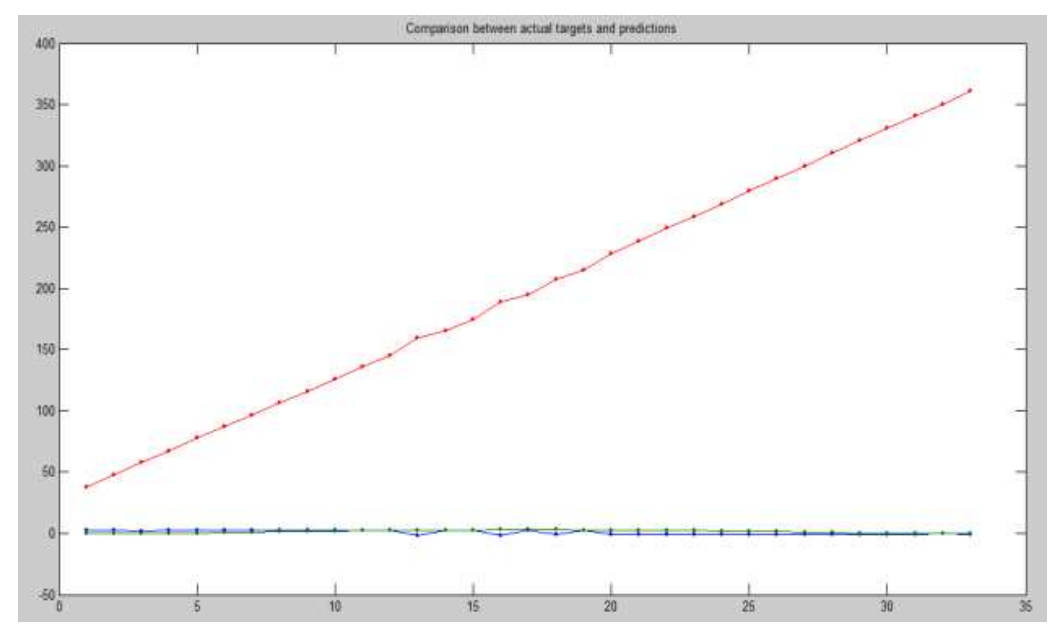

Figure 8. Comparisons between actual and predicted targets for inverse kinematics.

\section{CONCLUSION}

The differences in deduced and predicted values using ANN for 3-DOF robotic manipulator, shows that zero error is achieved by the proposed method. ANN can be trained so that fast and acceptable solutions are achieved. ANN can become an alternate method to map the forward and inverse kinematics solutions.

\section{REFERENCES}

[1] Saeed B. Niku, Introduction to Robotics: Analysis, Systems, Applications, Prentice Hall, p1-349. (2001)

[2] R.K. Mittal, I.J. NagrathRobotics and Control. New Delhi: Tata Mc Graw Hill Publishing Company Limited. ,(2003)

[3] S. K. Saha, Introduction to Robotics, Tata McGraw Hill, p1-425. ,(2008)

[4] M Gopal, Digital Control and State Variable Methods: Conventional and Intelligent Control Systems. (2009)

[5] Vijayant Agarwal, Ph. D. Thesis, Studies of Dynamics and Control of Robotic System using Soft Computing Techniques, University of Delhi. (2008)

[6] Allon Gurez ,Ziauddin Ahmad, Solution to the inverse kinematics problem in robotics by neural network., ICNN88. (1988),

[7] L.Acosta,G.N.Marichal,L.Moreno,J.J.Rodrigo,.Hmilton,J.A.Mendez, A robotic system based on neural network controllers.,artificial intelligence in engineering page 393-398. (1999)

[8] Vijyant Agarwal, A. P. Mittal, B. C. Nakra, A Study of Fuzzy Logic Based Inverse Kinematics Solution, Proceedings International Conference on Computer Applications in Electrical Engineering, Roorkee, p1-4. (2005),

[9] Srinivasan Alavander, M.J. Nigam, Inverse Kinematics Solution of 3DOF Planar Robot using ANFIS, International Journal of Computers, Communications and Control, Vol. III, p150-155. (2003),

[10] Jose Antonio Martin H. ,Javier de Lope, Matilde Santos A method to learn the inverse kinematics of multi-link robots by evolving neuro-controllers, Neurocomputing, 2806-2814. (2009). 


\section{BIOGRAPHIES OF AUTHORS}

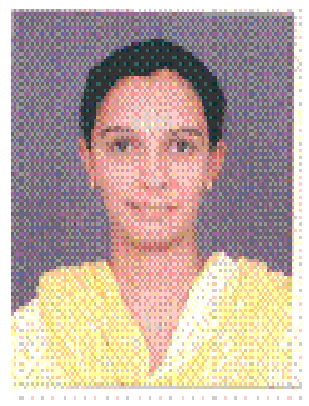

Ms. Jolly Shah is a Research Scholar at National Institute of Technology, Kurukshetra,India. She is Mechanical Engineering graduate from Birla Vishwakarma Mahavidyalaya, Vallabhvidyanagar, Gujarat, one of the premier institutes in the western India. She has obtained his M.E. degree in the field of Machine Design with Gold Medal and topper in university from Birla Vishwakarma Mahavidyalaya, Vallabhvidya nagar, Gujarat in the year 2005. Her area of research is Robotics. She has published 9 papers in conferences. She is associated with the Institution of Engineers (India) and Indian Society of Technical Education (ISTE).

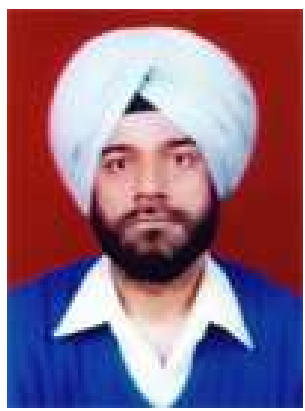

Dr. S.S.Rattan is a professor and former head of the Mechanical department at National Institute of Technology, Kurukshetra, India. His area of interest are Tribology, Fluid Engineering \& Machine Design. He received "The Sir Rajendra Nath MookerjeeMemorial Prize" for the best paper in the Journal Of Institute Of Engineers (Mech.Division) for year 2002. He is also Author of books of Theory of Machines (TMH) \& Fluid Mechanics and Machinery (Khanna Publishers). Total no. of Research Publications: 29

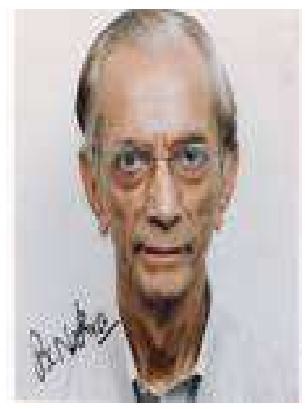

Professor B.C.Nakra worked as a Professor in Mechanical Engineering at Indian Institute of Technology, Delhi for several years before joining ITM Gurgaon in 2006. At IIT Delhi, he held B.H.E.L. Chair and Ram Rajindra Malhotra Emeritus Chair, in addition to having served as Head of Mechanical Engineering Dept., Head and joint Professor, Industrial Tribology, Machine Dynamics and Maintenance Engineering Centre and Head Instrument Design \& Development Centre .He was Dean, U.G. studies, Deputy Director (Faculty) and Offg. Director at IIT Delhi. During 2001 -2003, he also worked as Professor of Eminence at Netaji Subhash Institute of Technology, Delhi University. Presently, he is Professor of Eminence at ITM University, Gurgaon.He has been involved in teaching and research in the areas of Vibration control and analysis of machines and structures, Condition monitoring and diagnostic maintenance, Dynamic design, Mechatronics, Instrumentation and Automatic Controls. He has guided $21 \mathrm{Ph}$. Ds , 67 M.Tech thesis and has published 3 books, 174 research papers in reputed journals and Conference Proceedings, in addition to 68 technical reports . Based on his research contributions, he has been elected Fellow of Indian National Science Academy (FNA), Indian National Academy of Engineering (FNAE), Academy of Sciences (F.A.Sc) National Academy of Sciences (FNASc.) and of World Innovation Foundation (FWIF). He has received awards like C.V.Raman award of Acoustical Society of India, Eminent Engineer award of Institution of Engineers India, G.P. Chatterjee Memorial award from INSA, Distinguished Mechanical Engineering Educator from ISME etc. He has served on the editorial board of Sadhana, Journal, PINSA, Journal, Tribology International, Maintenance Journal, International Journal of Applied Maths and Mechanics.He has been consultant to leading industries and organizations including B.H.E.L., Indian Railways, ONGC, SAIL, EIL, BEL, Eicher, Escorts, Mahindra \& Mahindra, Hero Motors, NSTL etc. in addition to lecturing in continuing education programmes and has been involved in several sponsored research projects of DST, ARDB, CSIR and others. 